\title{
Attention enhances apparent perceptual organization
}

\author{
Antoine Barbot $^{1,2} \cdot$ Sirui Liu $^{1} \cdot$ Ruth Kimchi $^{3}$ - Marisa Carrasco ${ }^{1,4}$
}

Published online: 28 August 2017

(C) Psychonomic Society, Inc. 2017

\begin{abstract}
Perceptual organization and selective attention are two crucial processes that influence how we perceive visual information. The former structures complex visual inputs into coherent units, whereas the later selects relevant information. Attention and perceptual organization can modulate each other, affecting visual processing and performance in various tasks and conditions. Here, we tested whether attention can alter the way multiple elements appear to be perceptually organized. We manipulated covert spatial attention using a rapid serial visual presentation task, and measured perceptual organization of two multielements arrays organized by luminance similarity as rows or columns, at both the attended and unattended locations. We found that the apparent perceptual organization of the multielement arrays is intensified when attended and attenuated when unattended. We ruled out response bias as an alternative explanation. These findings reveal that attention enhances the appearance of perceptual
\end{abstract}

The original version of this article was revised.

Antoine Barbot and Sirui Liu have first co-authorship

Electronic supplementary material The online version of this article (https://doi.org/10.3758/s13423-017-1365-x) contains supplementary material, which is available to authorized users.

Marisa Carrasco

marisa.carrasco@nyu.edu

1 Department of Psychology, New York University, New York, NY, USA

2 Center for Visual Science, University of Rochester, Rochester, NY, USA

3 Department of Psychology and Institute of Information Processing and Decision Making, University of Haifa, Haifa, Israel

4 Center of Neural Science, New York University, New York, NY, USA organization, a midlevel vision process, altering the way we perceive our visual environment.

Keywords Voluntary attention · Perceptual organization . Appearance

Our world does not appear to us in terms of bits and pieces; instead, we see things as unified wholes, such as objects and surfaces. Perceptual organization is the process that structures the complex retinal inputs into perceptually coherent units-a crucial process for the further identification and recognition of objects and surfaces in the environment. Gestalt psychologists suggested that perceptual organization encompasses grouping and segregation processes, with a set of grouping principles that shape how we perceive multiple elements such as proximity, similarity, good continuation, closure, and more recently, common region and element connectedness (see reviews by Peterson \& Kimchi, 2013; Wagemans et al., 2012).

Selective attention is another key process that allows efficient and meaningful representation of our environment. Attention enables us to overcome the information overload our limited visual systems are confronted with by selecting and prioritizing the processing of relevant information, while the rest of information is processed to a lesser extent. In everyday life, we regularly deploy attention covertly, without moving our eyes, to monitor relevant locations. Spatial covert attention improves observers' performance in many discrimination, detection, and localization tasks (see reviews by Carrasco, 2011; Carrasco \& Barbot, 2015).

Importantly, attention does not only improve objective performance, it also alters the way information appears to us. Empirical studies have provided compelling evidence that attention alters the appearance of basic visual features of single, isolated objects, enhancing many dimensions, 
among others, perceived contrast (Anton-Erxleben, Abrams, \& Carrasco, 2010; Carrasco, Fuller, \& Ling, 2008; Carrasco, Ling, \& Read, 2004; Liu, Abrams, \& Carrasco, 2009; Störmer \& Alvarez, 2016; Störmer, McDonald, \& Hillyard, 2009), brightness (Tse, 2005), spatial frequency (Abrams, Barbot, \& Carrasco, 2010; Gobell \& Carrasco, 2005), size (Anton-Erxleben, Henrich, \& Treue, 2007), speed (Anton-Erxleben, Herrmann, \& Carrasco, 2013; Turatto, Vescovi, \& Valsecchi, 2007), flicker rate (Montagna \& Carrasco, 2006), and color saturation, but not hue (Fuller \& Carrasco, 2006).

Perceptual organization and selective attention are two crucial processes that shape the way we perceive our environment, but it is debated whether and how they influence each other. Traditional theories claimed that perceptual organization is preattentive, taking place automatically in a bottomup, stimulus-driven way (e.g., Julesz, 1981). However, perceptual organization and attention can mutually constrain and facilitate each other to yield coherent representations and meaningful percepts (review by Kimchi, 2009). For instance, perceptual organization constrains attentional selectivity, facilitating responses to two inputs when these belong to the same, rather than to different, objects (e.g., Duncan, 1984). Moreover, the mere organization by Gestalt factors of visual elements into an object automatically attracts attention (Kimchi, Yeshurun, \& Cohen-Savransky, 2007). Attention can also constrain perceptual organization, influencing target-flanker interaction (Freeman, Sagi \& Driver, 2001) and figure-ground assignment (Vecera, Flevaris, \& Filapek, 2004). Indeed, some, but not all, forms of grouping require attention (Ben-Av, Sagi, \& Braun, 1992; Kimchi \& Razpurker-Apfeld, 2004; Mack, Tang, Tuma, Kahn, \& Rock, 1992). Perceptual grouping of elements by proximity and/or similarity also interacts with the subjective attentional grouping induced by experimental instructions, modulating performance (Carrasco \& Chang, 1995). In sum, the relation between perceptual organization and attention is multifaceted, and whereas some forms of perceptual organization can occur without attention (Braun \& Sagi, 1991; Kimchi \& Peterson, 2008), attention can nevertheless modulate perceptual organization processes.

Here, we investigated whether and how attention alters the way we perceive the organization of visual scenes. Specifically, we tested the impact of spatial attention on the way we experience the perceptual organization of multiple visual elements - a mid-level visual function. We manipulated endogenous (voluntary) attention in a rapid serial visual presentation (RSVP) task, as in previous studies (Abrams et al., 2010; Liu et al., 2009). When participants had not detected an RSVP target, they performed an unrelated perceptual organization judgment task in which they compared the organization of two arrays of multiple elements perceptually grouped into columns/rows by luminance similarity. Considering that such grouping can occur under inattention (Kimchi \& RazpurkerApfeld, 2004; Russell \& Driver, 2005), any attentional influence on their apparent perceptual organization would be attributed to top-down attention modulations on perceived organization.

\section{Method}

\section{Participants}

Twenty subjects participated in this study (Experiment 1: twelve observers, age range: $20-24$ years; Experiment 2: eight observers, age range: $21-31$ years). For each experiment, all participants, except one author (S.L. in Experiment 1 and A.B. in Experiment 2), were naïve to the purpose of the study and had normal or corrected-to-normal vision. Six out of $12 \mathrm{ob}-$ servers in Experiment 1, and three out of 8 observers in Experiment 2, were trained psychophysical observers. Participants signed an informed consent approved by the NYU Institutional Review Board and received a compensation of $\$ 10 /$ hour.

\section{Apparatus}

Stimuli were generated using MATLAB (MathWorks, Natick, MA) and Psychtoolbox (Kleiner, Brainard \& Pelli, 2007) and were displayed in a dark room on a calibrated 22-in. CRT monitor $(1280 \times 960,85 \mathrm{~Hz})$ placed $57 \mathrm{~cm}$ from the observer. To ensure that participants maintain fixation during the experiment, their heads were stabilized on a chin rest, and eye fixation was monitored online using an eye-tracking system (Eyelink 1000, SR Research).

\section{Stimuli}

A fixation point ( $1^{\circ}$ visual angle [dva] diameter) was presented centrally on a gray background throughout the experiment. RSVP stimuli were composed of black letters $(1 \times 1 \mathrm{dva}$; any letter from the alphabet) presented at 25 possible positions separated by 1 dva on a $5 \times 5$ location grid centered at \pm 7 dva horizontally from both sides of fixation. Perceptual organization stimuli were 25 black and white dots ( 0.4 dva diameter each; $85 \%$ luminance contrast between black and white dots) at each position of the $5 \times 5$ grid that were more or less organized either as rows or columns (see Fig. 1a). Each stimulus was created by combining two reference stimuli: a perfectly organized stimulus (either as rows or columns) and a disorganized stimulus, with respect to the row/column organization of interest. The level of organization was manipulated by varying the probability that elements of the grid were drawn either from the organized or from the disorganized stimulus configuration (see Fig. S1a in the Supplementary 

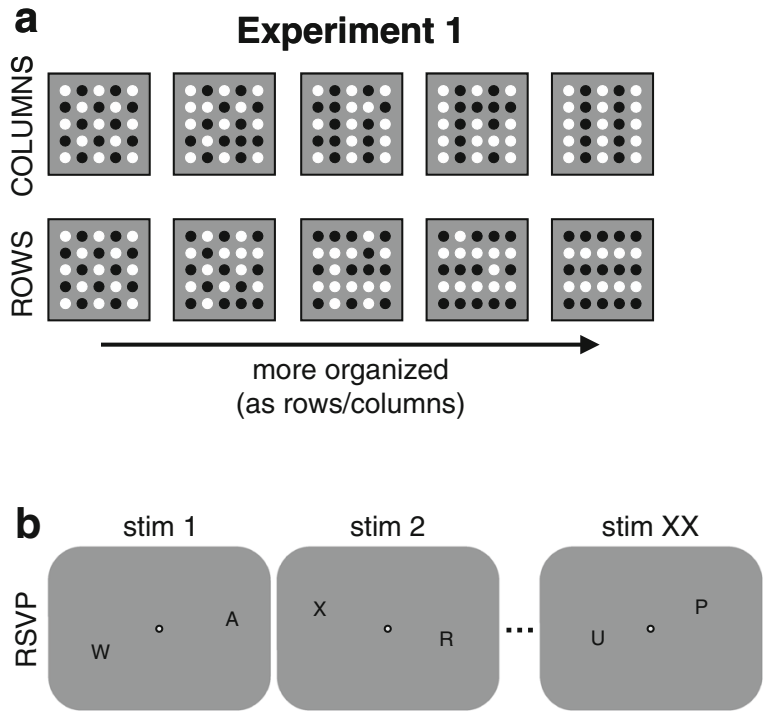

\section{Experiment 2}
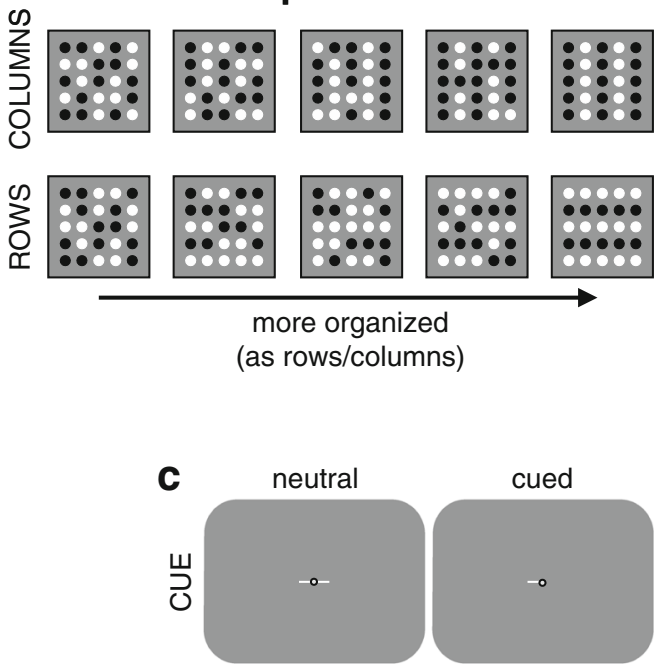

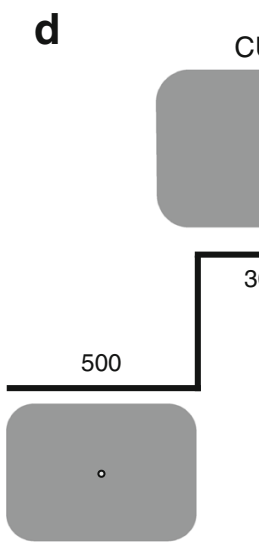

fixation
CUE

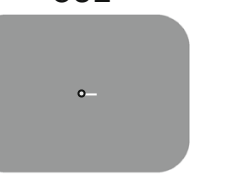

300

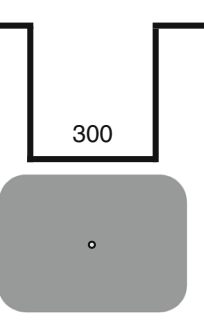

delay

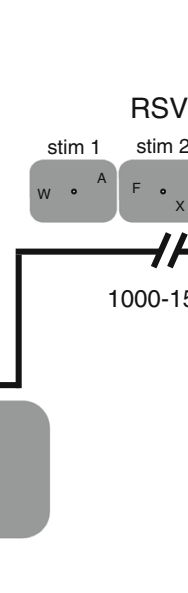

\section{STIMU}
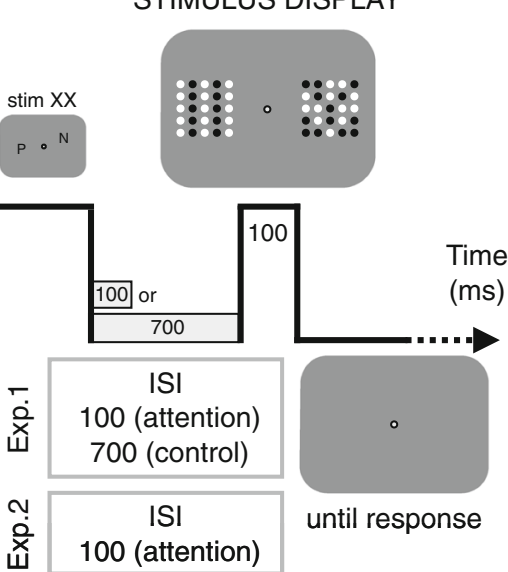

RSVP task

Press SPACE if you see a target ' $X$ ' letter

\section{SPACE}

- otherwise -

Appearance task

Which stimulus was more (Exp.1) or less (Exp.2) organized?

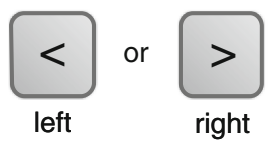

Fig. 1 Experimental procedure. a Perceptual organization stimuli were organized by luminance similarity into columns or rows. The organization level could go from being disorganized in terms of row/ column (Experiment 1: checkerboard; Experiment 2: random organization) to a fully organized stimulus. b RSVP sequences were made of a series of letters presented randomly at one of 25 possible locations $(5 \times 5$ grid). $\mathrm{c}$ Attention cues were central cues indicating with $100 \%$ validity either one (cued) or both (neutral) RSVP streams to attend to.

Material). In Experiment 1, checkerboards were used as disorganized stimuli, which although organized themselves are by definition totally disorganized in terms of row/columns. A more monotonic manipulation of perceptual organization was used in Experiment 2, using randomly organized stimuli as the disorganized configuration (see Fig. S1b in the Supplementary Material for examples of stimuli at all organization levels). In each trial, one stimulus was the test stimulus, with one of nine possible noise levels $(0 \%, 12.5 \%$, $25 \%, 37.5 \%, 50 \%, 62.5 \%, 75 \%, 87.5 \%, 100 \%$ ) and the other stimulus was the standard stimulus (fixed, intermediate noise level, $50 \%$ organized as rows/columns). Both stimuli were d Trial sequence began with a fixation period followed by a central cue. Then, RSVP letter streams were presented on both sides of fixation, and participants had to detect a target letter $X$. If they did not report the presence of a RSVP target, they had to report which of two subsequent stimuli was more organized. The RSVP sequence and the onset of the perceptually organized stimulus display were separated by either a short $(100 \mathrm{~ms}$; Experiments 1 and 2) or a long (700 ms; Experiment 1) ISI. Trial sequence was contingent on fixation using online eye tracking

organized into rows or columns, which was randomly determined on a trial-by-trial basis.

\section{Procedure}

Figure $1 \mathrm{~d}$ shows a sample trial sequence. Each trial was divided in two parts: RSVP and perceptual organization tasks. First, participants were asked to detect a target letter $(X)$ presented within one of two RSVP letter streams. Each RSVP stream was composed of different letters presented at random positions within the predefined grid, ensuring that spatial attention was deployed across a relatively large area (see Fig. 
1b). The trial started with a fixation period and was followed by a central attentional cue (see Fig. 1c). In two thirds of the trials (valid trials), the attentional cue was a horizontal line ( 0.75 dva length) next to fixation indicating with $100 \%$ validity the side (left or right) of the relevant, upcoming RSVP stream that could contain the target letter, encouraging participants to attend covertly toward that side. On the remaining one third of the trials (neutral trials), the cue consisted of two horizontal lines pointing toward both RSVP streams, indicating that the target would be equally likely to be presented in any of the two RSVP streams. After a brief delay, the two RSVP streams were presented simultaneously. To ensure that observers focused their attention during the entire RSVP sequence, the total duration of the RSVP sequence alternated randomly from trial to trial, lasting $1,1.25$, or 1.5 seconds. Participants were asked to press the space bar if they detected the target letter $X$ and to ignore the subsequent stimulus presentation; otherwise, they were asked to do the second part of the trial; i.e., regardless of whether their response was a "miss" (they missed the presence of a target), or a "correct rejection" (they correctly noticed the absence of a target). The target letter was presented on $25 \%$ of the trials to ensure that consistent voluntary attention was engaged. RSVP difficulty was manipulated by increasing or decreasing the number of letters within a single RSVP interval (i.e., by manipulating the RSVP letter duration). For each participant, the RSVP rate was determined during practice at the beginning of the first experimental session using an adaptive staircase procedure and adjusted between experimental blocks to maintain a constant difficulty level (overall $d^{\prime}$ of $\sim 1.5$ ). RSVP rates at which observers performed the task ranged from 8 to 40 letters/second (average: $22.3 \pm 5.9$ letter/s) during the RSVP interval.

Following the RSVP task, two arrays of $5 \times 5$ dots patterns - the standard and test stimuli-were shown simultaneously for $100 \mathrm{~ms}$ on both sides of fixation. Participants were asked to compare both arrays and report as accurately as possible which array (left or right) was more organized, by pressing the corresponding keyboard key ( $<$ for left, $>$ for right). Critically, to rule out response bias, we took advantage of the known temporal dynamics of voluntary spatial attention, which takes $\sim 300 \mathrm{~ms}$ to be covertly redeployed (e.g., Nakayama \& Mackeben, 1989; Posner, 1980) by manipulating the interstimulus interval (ISI) between the RSVP and the perceptual organization task. In experimental blocks, a 100-ms ISI ensured that participants had not had enough time to voluntarily reallocate their spatial attention to both upcoming stimulus locations; attention would still be at the attended RSVP location side during the presentation of the perceptually organized stimuli. Conversely, in control blocks, a 700ms ISI provided enough time to redistribute attention equally across both locations.
Participants could only deploy their covert attention during the task. Trial sequence was contingent on fixation and online eye tracking ensured that participants were fixating the center of the screen during the entire trial sequence. If participants broke fixation (define by a 1.5 dva radius area around fixation), the trial was aborted and reran later during the session. Participants were told that the two tasks were totally independent (i.e., the cues only carried information about the RSVP task regardless of the side with the higher perceptual organization stimulus). Thus, participants should monitor both stimuli in the second part to judge effectively which one is more perceptually organized. This disguised the true dimension of interest - perceived organization - to avoid the possibility of participants giving more weight to the attended location when comparing the two stimuli in the judgment task.

In Experiment 2, the procedure was identical to that of Experiment 1, except that participants were asked to report the stimulus that was less perceptually organized and only the 100-ms ISI condition was used. Reverse instructions have been shown to efficiently control for response biases (e.g., Anton-Erxleben et al., 2007; Carrasco et al., 2004; Fuller \& Carrasco, 2006; Liu et al., 2009; Störmer \& Alvarez, 2016), as enhanced perceived organization due to attention or to response biases would result in opposite effects.

In Experiment 1, observers participated in five experimental sessions, each divided in two counterbalanced sets of 270 trials per ISI condition, with breaks every 90 trials. Thus, each participant completed a total of 2,700 trials, with 1,350 trials per ISI condition. In Experiment 2, only the short (100 ms) ISI condition was used and observers participated in six experimental sets of 270 trials, for a total of 1,620 trials. At the end of each 90-trials block, the participant's RSVP detection performance (\% correct and corresponding $d^{\prime}$, as well as hit and false-alarm rates) was displayed on the screen.

\section{Analysis}

RSVP detection sensitivity $\left(d^{\prime}\right)$ was computed for each participant independently from the participant response criterion. Group-averaged $d^{\prime}$ values for cued and neutral conditions were calculated for the main and control ISI conditions to assess the effect of attention on RSVP detection performance. A repeated-measures ANOVA and post hoc, paired $t$ tests were used to examine whether attention was effectively manipulated in the RSVP task, similarly for both ISI conditions. For the organization judgment task, we calculated the percentage of trials on which the observer chose the test stimulus as being more organized than the standard stimulus, as a function of the organization level of the test stimulus. We fitted the data using a Weibull psychometric function:

$\psi=\gamma+(1-\gamma-\lambda)\left\{1-\exp \left[-\left(\frac{x}{\alpha}\right)^{\beta}\right]\right\}$ 
in which $\psi$ is the proportion of trials the test stimulus was chosen over the standard stimulus; $x$ is the organization noise level of the test stimulus; $\alpha$ is the location parameter; $\beta$ is the slope; and $\gamma$ and $\lambda$ are the lower and upper asymptotes respectively, using maximum likelihood procedure. The point of subjective equality (PSE) - the point at which the test and standard stimuli are subjectively equal and equally likely to be chosen as more organized-were then interpolated from individual function fits to each condition. Note that although PSE estimates and $\alpha$ parameters are both indicative of horizontal shifts of the function, they are not the same. In Experiment 2 (reverse instructions), the organization noise level of the test stimulus $(x)$ was reversed to go from $100 \%$ to $0 \%$, such that Weibull functions could be fitted and depict the increasing proportion of trials for which the test stimulus was chosen over the standard stimulus. Repeated-measures ANOVAs and post hoc, paired $t$ tests were used to examine the effects of cueing (test, standard, or both cued) and ISI (main or control) on the PSE estimates. In all cases in which Mauchly's test of sphericity indicated a violation of the sphericity assumption, Greenhouse-Geisser-corrected values were used. Additional analyses on the Weibull parameters were also performed (see Supplementary Material).

\section{Results}

\section{RSVP performance}

Figure 2 shows the averaged RSVP detection performance $\left(d^{\prime}\right)$ values for the neutral and cued conditions. In Experiment 1 (see Figs. 2a-b), a two-way repeated-measures ANOVA showed a significant main effect of cueing on RSVP detection performance, $\left.F(1,11)=29.16, p<.001, \eta_{\mathrm{p}}{ }^{2}=.73\right]$ no main effect of ISI, $F(1,11)<1$, and no interaction between cueing and ISI
$F(1,11)<1$. Post hoc paired $t$ tests showed that RSVP performance was significantly better for the cued than for the neutral condition in both ISI conditions (100-ms ISI: $t(11)=4.14, p=$ .0016 , Cohen's $d=1.20$; 700-ms ISI: $t(11)=4.53, p<.001$, Cohen's $d=1.31$. Similarly, in Experiment 2 (see Fig. 2c) attention improved RSVP detection performance, $F(1,7)=91.28$, $p<.001, \eta_{\mathrm{p}}{ }^{2}=.93 ; t(7)=9.55, p<.001$, Cohen's $d=3.52$.

\section{Perceived organization judgment}

Figure 3 show group-averaged appearance judgment values and corresponding fitted curves for both Experiment 1 (see Fig. 3a-b) and Experiment 2 (Fig. 3c). In Experiment 1, we found that in the 100-ms ISI condition (see Fig. 3a) the curve for the test-cued condition shifted to the left relative to the neutral condition, indicating that observers perceived the test as being more organized when it was cued relative to the neutral attention condition. Conversely, when the standard stimulus was cued, the curve shifted to the right relative to the neutral condition, indicating that the test was perceived to be less organized when unattended. However, for the long ISI condition (see Fig. 3b), differences among the three cueing conditions disappeared, indicating that the cueing effects in the experimental (100-ms ISI) condition were not due to a response bias toward the cued side (i.e., participants would have been more likely to report the cued location regardless of how they had perceived the two stimuli).

Experiment 2-reverse instructions - provided direct evidence that the results from Experiment 1 were not due to response biases. When participants were asked to judge the less organized stimuli (see Fig. 3c), the test-cued curve shifted to the left compared to the neutral condition, indicating that the test was less likely to be reported as the least organized stimulus when attended. Conversely, when the standard stimulus was cued, the curve shifted to the right, indicating that the a

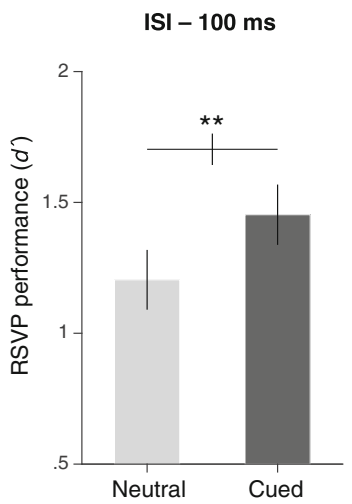

Experiment 1

$\mathrm{N}=12$ b
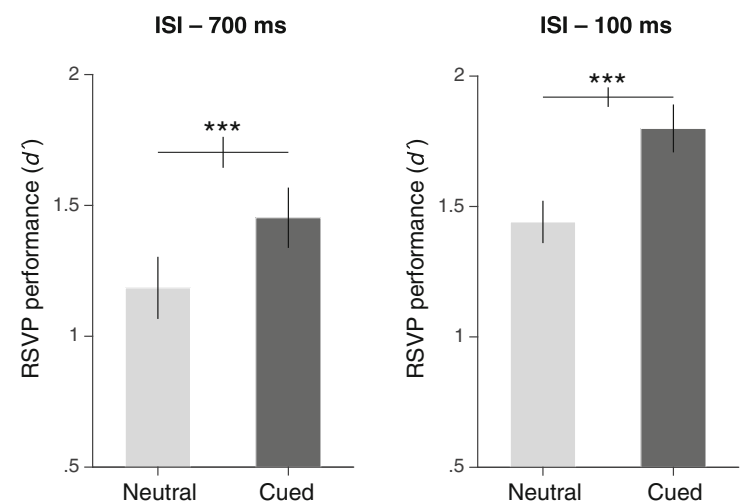

Fig. 2 RSVP performance. Detection sensitivity $(d)$ was higher when the side that could contain the RSVP target was precued relative to the neutral condition for both Experiment 1 (a: attention, short ISI; b:

control, long ISI) and Experiment 2 (c: attention, reverse instructions). Error bars represent $\pm 1 S E M * p<.05$. ** $p<.01$. *** $p<.001$ 
a Experiment 1- Attention (short ISI - $100 \mathrm{~ms}$ )
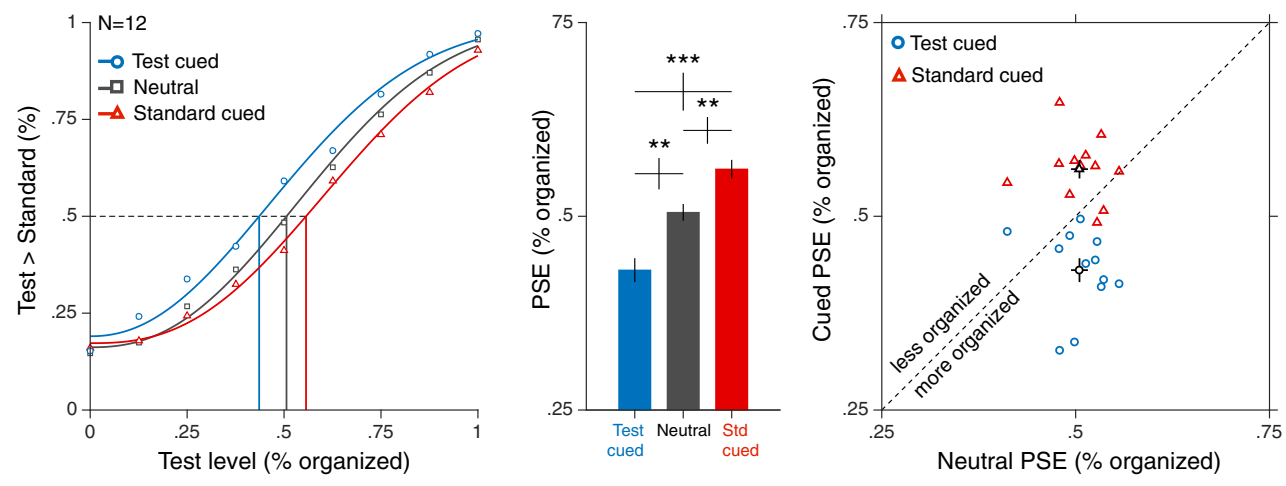

b Experiment 1 - Control (long ISI - $700 \mathrm{~ms}$ )
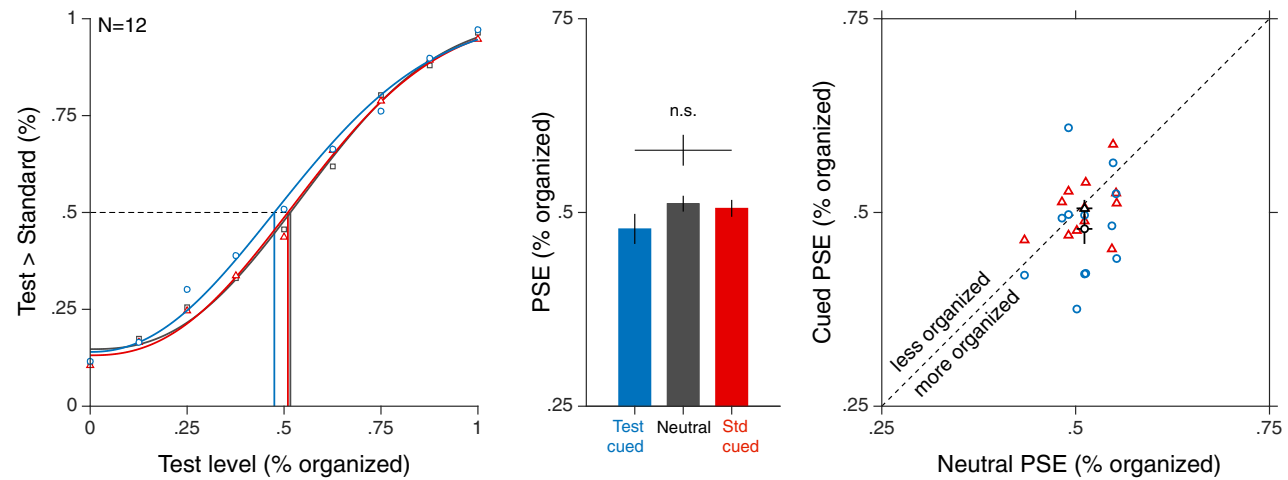

\section{Experiment 2 -Attention: reverse instructions (short ISI - $100 \mathrm{~ms}$ )}

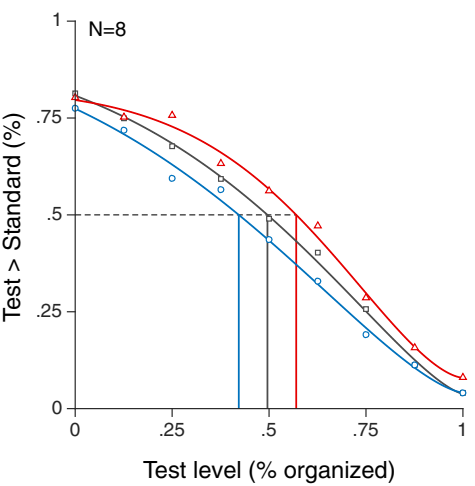

Fig. 3 Perceived organization appearance. Results are shown for Experiment 1 (a: attention, short ISI; b: control, long ISI) and Experiment 2 (c: attention, reverse instructions). Left column: Average percentage of trials on which participants perceived the test to be more organized than the standard stimulus as a function of the test organization

test was more likely to be reported as being less organized when unattended. This pattern of results is consistent with the idea that the grid of dots appeared to be more organized when attended. Critically, response biases would have resulted in the opposite pattern, with the cued stimuli being more likely to be selected regardless of the instructions. Of note, no difference in response time pattern with attention was observed between Experiment 1 (report the "more" organized stimulus;
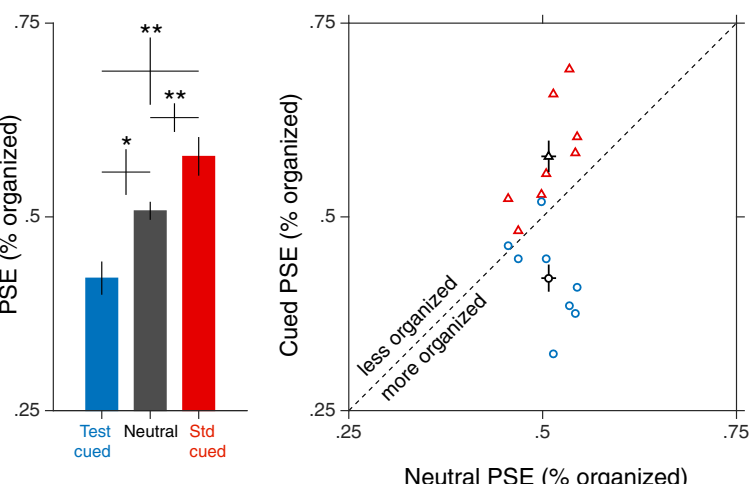

level. Middle column: Group-average of the point-of-subjective-equality (PSE) estimates for each cueing condition; Right column: Individual PSEs for the test-cued and standard-cued conditions as a function of individual PSEs in the neutral condition. Error bars represent $\pm 1 S E M$. $* p<.05 . * * p<.01 . * * * p<.001$. (Color figure online)

for 100-ms ISI only) and Experiment 2 (report the "less" organized stimulus); i.e., no interaction between cueing and experimental instructions: $\mathrm{F}(2,36)=1.11, p=.341$. Thus, these results ruled out response biases explanation and further confirmed that perceived perceptual organization was enhanced at the attended location.

The average of individual PSE values for each cuing condition in Experiment 1 (see Fig. 3a-b) and Experiment 2 (Fig. 
$3 c)$ conditions were consistent with these results. In Experiment 1, a two-way repeated-measures ANOVA on PSEs was used to test whether and how cueing and ISI affected perceived organization. There was a significant main effect of cueing on PSEs, $F(2,22)=13.11, p<.001, \eta_{\mathrm{p}}{ }^{2}=.54$, and an interaction between cueing and ISI on PSEs, $F(2,22)=$ $10.76, p<.001, \eta_{\mathrm{p}}{ }^{2}=.50$. One-way ANOVAs showed a significant effect of cueing on PSEs in the 100-ms ISI condition, $F(2,22)=19.70, p<.001, \eta_{\mathrm{p}}{ }^{2}=.64$, reflecting significant differences among the three cueing conditions, test-cued vs. neutral: $t(11)=3.69, p=.0036$, Cohen's $d=$ 1.08; standard-cued vs. neutral: $t(11)=3.24, p=.0078$, Cohen's $d=.94$; test-cued vs. standard-cued: $t(11)=5.33, p$ $<.001$, Cohen's $d=1.54$. In contrast, for the 700-ms ISI condition, no significant effect of cueing was observed, $F(2$, $\left.22)=1.94, p=.168, \eta_{\mathrm{p}}{ }^{2}=.15\right]$. Similarly, in Experiment 2 (see Fig. 3c), attention significantly modulated PSEs when a 100 -ms ISI was used, $F(1.13,7.91)=12, p=.008, \eta_{\mathrm{p}}{ }^{2}=.63$, with all three cueing conditions differing from each other, testcued vs. neutral: $t(7)=2.95, p=.0215$, Cohen's $d=1.06$; standard-cued vs. neutral: $t(7)=3.79, p=.0068$, Cohen's $d=$ .1.80; test-cued vs. standard-cued: $t(7)=3.62, p=.0085$, Cohen's $d=1.28$. Of note, additional analyses on Weibull parameters (see Fig. S2) showed significant changes in Weibull thresholds $(\alpha)$ for Experiment 1 (100-ms ISI) and Experiment 2 (100-ms ISI, reversed instructions), but not for the long ISI condition (Experiment 1, 700-ms ISI), consistent with the changes in PSEs and horizontal shifts of the functions we observed.

Individual PSEs for the test-cued and standard-cued conditions as a function of corresponding individual PSEs in the neutral condition are shown in the three right panels of Fig. 3. When a short 100-ms ISI was used (Fig. 3a, c), regardless of the direction of the instruction, most observers' PSEs were lower for the test-cued condition than for their corresponding neutral condition, and higher for the standard-cued condition than for their corresponding neutral condition. In contrast, when a longer ISI was used (Fig. 3b), observers had enough time to redeploy their attention to both stimulus locations and PSEs in both test-cued and standard-cued conditions were intermingled and closely around the neutral line, consistent with no effect of cueing on perceived organization. Figure 4 shows a schematic representation of the changes in perceived organization with attention in Experiment 1 (short ISI: testcued $=43 \%$, standard-cued $=56 \%$ ) and Experiment 2 (testcued $=42 \%$, standard-cued $=58 \%$ ).

\section{Discussion}

Our results reveal that attention alters the appearance of midlevel visual representations; it enhances the perceived organization of multiple elements at the attended location similar to the way it alters the appearance of early visual features of single isolated stimuli. When attention was at the test stimulus location, a less organized test stimulus was sufficient to match a more organized standard stimulus. Conversely, when the standard stimulus was attended, higher organization was required to subjectively match the standard stimulus. Such shifts in PSE values are consistent with enhanced perceived organization with attention.

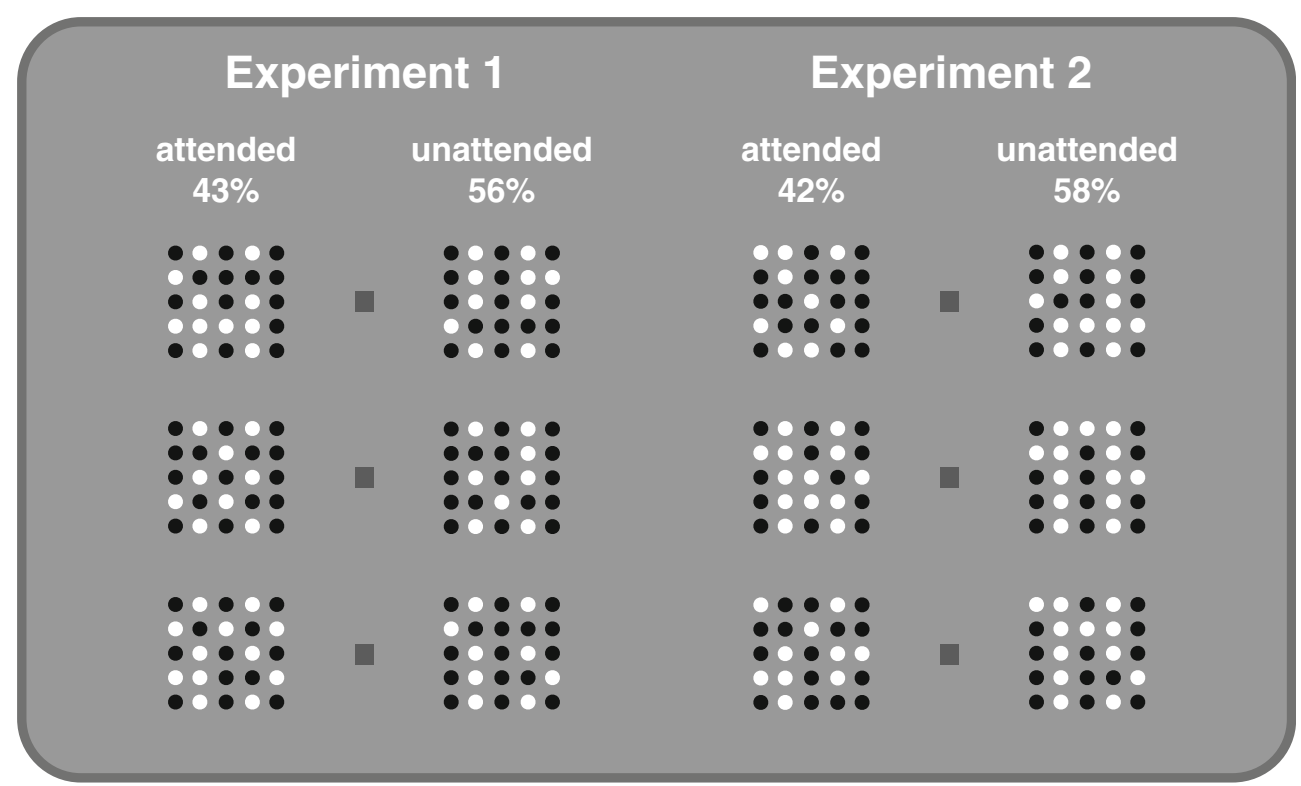

Fig. 4 Schematic representations of the changes in apparent perceptual organization with attention in Experiment 1 (left panel: attention, short ISI) and Experiment 2 (right panel: reverse instructions). When attended, the less organized stimuli on the left columns of each panel appeared as organized as the unattended stimuli that were more organized on the right columns of each panel 
One concern is that participants could simply weight the cued location more in their decision, regardless of the perceived organization. Several experimental controls for response bias have been conducted to demonstrate that attention does alter appearance. Here, we adapted a protocol used in previous studies (Abrams et al., 2010; Liu et al., 2009) by combining two independent tasks: an RSVP task, in which endogenous attention is manipulated, and a perceptual organization comparison task, in which the optimal strategy would be to attend to both sides equally. The rationale of using two separate, unrelated tasks is that we could effectively control for response bias from adopting possible cue-related strategies for the judgment task while assessing separately that attention was effectively manipulated in the RSVP task. The optimal strategy for the comparison task would be to attend to both sides equally. Expectedly, when enough time was given to the observers to redeploy their attention to both stimulus locations, no difference in perceived organization was found, ruling out response bias as a possible explanation. Such control by lengthening ISI has been successfully used in previous studies on the effects of exogenous (Carrasco et al., 2004; Fuller, Rodriguez, \& Carrasco, 2008; Ling \& Carrasco, 2007; Störmer \& Alvarez, 2016) and endogenous (Abrams et al., 2010) attention on appearance. Moreover, the fact that this pattern of results was found regardless of the instructions (i.e., report the more or the less organized stimulus) provides evidence that observers did perceive the attended stimulus as being more perceptually organized and rules out response biases as a possible explanation. Other experimental controls have also been used to rule out cue bias, response bias and other alternative interpretations (e.g., using postcues instead of precues; for review, see Carrasco, 2009).

Previous research has focused on the effect of attention on the appearance of early visual features (e.g., perceived contrast or spatial frequency), which are likely to reflect modulations in early stages of processing (i.e., primary visual cortex). A recent study has shown that such low-level effects (i.e., increased perceived contrast) can impact the appearance of higher-level objects, such as enhanced facial attractiveness (Störmer \& Alvarez, 2016). However, such low-level effects are unlikely to explain changes in perceived organization, as perceived contrast is minimally altered by attention at the high suprathreshold luminance contrast (85\%) used for our stimuli (Cutrone, Heeger, \& Carrasco, 2014).

Visual scenes are not composed of single objects and are often cluttered, containing multiple elements that need to be segmented into coherent regions before further processing. Whereas single properties are represented relatively early, more complex visual attributes such as textural information and other forms of perceptual organization are represented at more intermediate processing stages, such as V2 and downstream areas (see review by Landy, 2013). Thus, the increase in perceived organization with attention may likely arise from attentional modulations at midlevel processing stages, such as enhanced second-order texture sensitivity (Barbot, Landy, \& Carrasco, 2011, 2012; Yeshurun \& Carrasco, 2000). Altered perceived organization may result from increased input response baseline of neural population at midlevel processing stages, similar to the effects of attention on apparent contrast at early processing stages (Cutrone et al., 2014). Overall, the fact that attention both improves performance and alters appearance of multiple visual dimensions (but not all; e.g., Fuller \& Carrasco, 2006) suggests a strong link between the attentional mechanisms improving visual processing and the concomitant effects on appearance.

Whereas traditional theories claimed that perceptual organization takes place preattentively, there is a strong interaction between attention and perceptual organization (see review by Kimchi, 2009). Attention can modulate intermediate stages of processing, affecting performance in various midlevel tasks, such as texture segregation and figure-ground segmentation (e.g., Barbot \& Carrasco, 2017; Barbot et al., 2011, 2012; Casco et al., 2005; Vecera et al., 2004; Yeshurun \& Carrasco, 2000; Yeshurun, Montagna \& Carrasco, 2008), both of which were first considered to occur preattentively (Julesz, 1981). The present findings further demonstrate that voluntary spatial attention modulates perceptual grouping of elements by luminance similarity, which can take place without attention (Kimchi \& Razpurker-Apfeld, 2004).

Traditional theories treated perceptual organization as a unitary phenomenon that operates at a single, early stage, in an automatic fashion. A growing body of research has challenged this traditional view, suggesting that perceptual organization is not a monolithic entity but rather represents a confluence of multiple processes that vary in time course, developmental trajectory, and attentional demands (see reviews by Kimchi, 2009; Wagemans et al., 2012). Neurophysiological and patient studies also support a multiplicity of neural processes, with different cortical mechanisms underlying different Gestalt grouping operations (Behrmann \& Kimchi, 2003; Han, Jiang, Mao, Humphreys, \& Gu, 2005). These studies suggest that at least two differential but correlated neural mechanisms of perceptual organization might coexist: one subserving more primitive low-level grouping, whereas the other, higher along the visual processing stream, underlying shape formation and higher-level configuration. Further investigations should evaluate whether and how attention affects distinct forms of perceptual organization.

To conclude, the present study revealed that attention alters the perceived organization of multiple visual elements, furthering our understanding of the way attention modulates midlevel processing stages and impacts visual appearance. Overall, these findings advance our knowledge of the relation between attention and perceptual organization, two selective and prioritizing mechanisms that improve visual processing and shape the way we experience our visual world. 
Acknowledgements This research was supported by NIH R01EY016200 to MC. We thank members of the Carrasco Lab for useful discussions.

\section{References}

Abrams, J., Barbot, A., \& Carrasco, M. (2010). Voluntary attention increases perceived spatial frequency. Attention, Perception \& Psychophysics, 72, 1510-1521.

Anton-Erxleben, K., Abrams, J., \& Carrasco, M. (2010). Evaluating comparative and equality judgments in contrast perception: Attention alters appearance. Journal of Vision, 10(11), 6.

Anton-Erxleben, K., Henrich, C., \& Treue, S. (2007). Attention changes perceived size of moving visual patterns. Journal of Vision, 7(11), 5.1-9.

Anton-Erxleben, K., Herrmann, K., \& Carrasco, M. (2013). Independent effects of adaptation and attention on perceived speed. Psychological Science, 24(2), 150-159.

Barbot, A., \& Carrasco, M. (2017). Attention modifies spatial resolution according to task demands. Psychological Science, 28(3), 285-296.

Barbot, A., Landy, M. S., \& Carrasco, M. (2011). Exogenous attention enhances 2nd-order contrast sensitivity. Vision Research, 51, 1086-1098.

Barbot, A., Landy, M. S., \& Carrasco, M. (2012). Differential effects of exogenous and endogenous attention on second-order texture contrast sensitivity. Journal of Vision, 12(8). https://doi.org/10.1167/12.8.6

Behrmann, M., \& Kimchi, R. (2003). What does visual agnosia tell us about perceptual organization and its relationship to object perception? Journal of Experimental Psychology: Human Perception \& Performance, 29(1), 19-42.

Ben-Av, M. B., Sagi, D., \& Braun, J. (1992). Visual attention and perceptual grouping. Perception \& Psychophysics, 52(3), 277-294.

Braun, J., \& Sagi, D. (1991). Texture-based tasks are little affected by second tasks requiring peripheral or central attentive fixation. Perception, 20(4), 483-500.

Carrasco, M. (2009). Attention: Psychophysical approaches. In T. Bayne, A. Cleeremans, \& P. Wilken (Eds.), Oxford companion to consciousness. Oxford: Oxford University Press.

Carrasco, M. (2011). Visual attention: The past 25 years. Vision Research, $51,1484-1525$.

Carrasco, M., \& Barbot, A. (2015). How Attention Affects Spatial Resolution. Cold Spring Harb Symp Quant Biol, 79, 149-160.

Carrasco, M., \& Chang, I. (1995). The interaction of objective and subjective organizations in a localization search task. Perception \& Psychophysics, 57(8), 1134-1150.

Carrasco, M., Fuller, S., \& Ling, S. (2008). Transient attention does increase perceived contrast of suprathreshold stimuli: A reply to Prinzmetal, Long, and Leonhardt (2008). Perception \& Psychophysics, 70(7), 1151-1164.

Carrasco, M., Ling, S., \& Read, S. (2004). Attention alters appearance. Nature Neuroscience, 7(3), 308-313.

Casco, C., Grieco, A., Campana, G., Corvino, M. P., \& Caputo, G. (2005). Attention modulates psychophysical and electrophysiological response to visual texture segmentation in humans. Vision Research, 45, 2384-2396.

Cutrone, E. K., Heeger, D. J., \& Carrasco, M. (2014). Attention enhances contrast appearance via increased input baseline of neural responses. Joural of Vision, 14(14), 16.

Duncan, J. (1984). Selective attention and the organization of visual information. Journal of Experimental Psychology: General, 113(4), 501-517.

Freeman, E., Sagi, D., \& Driver, J. (2001). Lateral interactions between targets and flankers in low-level vision depend on attention to the flankers. Nature Neuroscience, 4(10), 1032-1036.

Fuller, S., \& Carrasco, M. (2006). Exogenous attention and color perception: Performance and appearance of saturation and hue. Vision Research, 46(23), 4032-4047.
Fuller, S., Rodriguez, R. Z., \& Carrasco, M. (2008). Apparent contrast differs across the vertical meridian: Visual and attentional factors. Journal of Vision, 8(1), 11-16.

Gobell, J., \& Carrasco, M. (2005). Attention alters the appearance of spatial frequency and gap size. Psychological Science, 16(8), 644-651.

Han, S., Jiang, Y., Mao, L., Humphreys, G. W., \& Gu, H. (2005). Attentional modulation of perceptual grouping in human visual cortex: Functional MRI studies. Human Brain Mapping, 25(4), 424-432.

Julesz, B. (1981). Textons, the elements of texture perception, and their interactions. Nature, 290, 91-97.

Kimchi, R. (2009). Perceptual organization and visual attention. Progress in Brain Research, 176, 15-33.

Kimchi, R., \& Peterson, M. A. (2008). Figure-ground segmentation can occur without attention. Psychological Science, 19(7), 660-668.

Kimchi, R., \& Razpurker-Apfeld, I. (2004). Perceptual grouping and attention: not all groupings are equal. Psychonomic Bulletin \& Review, 11(4), 687-696.

Kimchi, R., Yeshurun, Y., \& Cohen-Savransky, A. (2007). Automatic, stimulus-driven attentional capture by objecthood. Psychonomic Bulletin \& Review, 14(1), 166-172.

Kleiner, M., Brainard, D., \& Pelli, D. G. (2007). What's new in Psychtoolbox-3? Perception, 36(14), 1-16.

Landy, M. S. (2013). Texture analysis and perception. In J. S. Werner \& L. M. Chalupa (Eds.), The new visual neurosciences (pp. 639-652). Cambridge: MIT Press.

Ling, S., \& Carrasco, M. (2007). Transient covert attention does alter appearance: A reply to Schneider (2006). Perception \& Psychophysics, 69(6), 1051-1058.

Liu, T., Abrams, J., \& Carrasco, M. (2009). Voluntary attention enhances contrast appearance. Psychological Science, 20(3), 354-362.

Mack, A., Tang, B., Tuma, R., Kahn, S., \& Rock, I. (1992). Perceptual organization and attention. Cognitive Psychology, 24(4), 475-501.

Montagna, B., \& Carrasco, M. (2006). Transient covert attention and the perceived rate of flicker. Journal of Vision, 6(9), 955-965.

Nakayama, K., \& Mackeben, M. (1989). Sustained and transient components of focal visual attention. Vision Research, 29, 1631-1647.

Peterson, M. A., \& Kimchi, R. (2013). Perceptual organization. Oxford: Oxford University Press.

Posner, M. I. (1980). Orienting of attention. Quarterly Journal of Experimental Psychology, 32, 3-25.

Russell, C., \& Driver, J. (2005). New indirect measures of "inattentive" visual grouping in a change-detection task. Perception \& Psychophysics, 67(4), 606-623.

Störmer, V. S., \& Alvarez, G. A. (2016). Attention alters perceived attractiveness. Psychological Science, 27(4), 563-571.

Störmer, V. S., McDonald, J. J., \& Hillyard, S. A. (2009). Cross-modal cueing of attention alters appearance and early cortical processing of visual stimuli. Proceedings of the National Academy of Sciences of the United States of America, 106, 22456-22461.

Tse, P. U. (2005). Voluntary attention modulates the brightness of overlapping transparent surfaces. Vision Research, 45(9), 1095-1098.

Turatto, M., Vescovi, M., \& Valsecchi, M. (2007). Attention makes moving objects be perceived to move faster. Vision Research, 47(2), 166-178.

Vecera, S. P., Flevaris, A. V., \& Filapek, J. C. (2004). Exogenous spatial attention influences figure-ground assignment. Psychological Science, 15(1), 20-26.

Wagemans, J., Elder, J. H., Kubovy, M., Palmer, S. E., Peterson, M. A., Singh, M., \& von der Heydt, R. (2012). A century of Gestalt psychology in visual perception: I. Perceptual grouping and figureground organization. Psychological Bulletin, 138(6), 1172-1217.

Yeshurun, Y., \& Carrasco, M. (2000). The locus of attentional effects in texture segmentation. Nature Neuroscience, 3, 622-627.

Yeshurun, Y., Montagna, B., \& Carrasco, M. (2008). On the flexibility of sustained attention and its effects on a texture segmentation task. Vision Research, 48, 80-95. 